\title{
MEASURING THE EFFECT OF DISCOURSE CONNECTIVES ON DISCOURSE COMPREHENSION: A CAUTIONARY NOTE ON THE USE OF FREE RECALL*
}

\author{
Melinda Sinclair*** \\ 245 Forman Avenue \\ Toronto, Ontario M4S 2S4 \\ Canada \\ E-mail: 76075.345@compuserve.com
}

Introduction

Even a cursory look at handbooks of writing reveals that these handbooks regard the relationships between utterances, and the way these are signalled by the writer, as important elements in effective writing. One of the devices used for signalling relations between utterances are the so-called "discourse connectives", also called "(cohesive) conjunctions", after Halliday and Hasan (1976), or "discourse markers". These include words and phrases like so, after all, therefore, but, although, nevertheless.

There is a robust intuition amongst those who advise on effective writing, and indeed amongst writers themselves, that the use of discourse connectives to explicitly signal the relationships between utterances facilitates discourse comprehension. The general direction of the advice is captured by Roen (1984: 23) with the phrase "the-more-the-merrier", or what I will call "the-more-the-better". According to Roen (1984: 23), rhetoricians' advice on the use of discourse connectives boils down to "basically unrestricted advice that writers should use intersentential cohesive conjunctions (transition words) to produce more coherent discourse ..." A typical example is Williams (1985: 50), who declares that "In general, the greater the use of discourse markers, the more cohesive and hence the more readable a text will be". 
But what is the actual effect of discourse connectives on discourse comprehension? A considerable body of empirical research is aimed at finding the answer to this question (e.g. Marshall \& Glock, 1978; Meyer, Brandt \& Bluth, 1980; Irwin, 1980; Bridge \& Winograd, 1982; Roen, 1984; Geva \& Ryan, 1985; Spyridakis \& Standal, 1987; Zinar, 1990). As Zinar (1990: 182) rightly observes, "Experimental results in this area have varied greatly, reflecting wide disparities in materials, procedures, and subject groups." It has become clear that a whole range of text and reader variables are involved. Nevertheless, on the whole the evidence supports the belief that insofar as discourse connectives do make a difference, they make a positive difference. Or to paraphrase Spyridakis and Standal (1987: 294), these studies provide "ample reason to believe" that discourse connectives help, and "no reason to believe" that they hinder. This empirical research therefore seems to provide some support for the "the-more-the-better" view on the use of discourse connectives in writing.

One of the measures of discourse comprehension used in empirical inquiries into the effect of discourse connectives on comprehension is free recall (cf. e.g. Roen, 1984; Caron, 1987; Zinar, 1990). In essence, this calls for the subject to read through or listen to a passage, and then to recall as much as possible of the content of the passage. The recalled information is then quantified in some way. The amount of information recalled from a text is interpreted as a measure of discourse comprehension: The more information recalled, the better the comprehension, and vice versa. So, for instance, if the level of free recall for a passage containing a large number of discourse connectives is higher than for the same passage with fewer (or no) discourse connectives, this is taken as evidence for a facilitative effect of discourse connectives.

The latter use of the measure of free recall to assess the effect of connectives on discourse comprehension forms the focus of this article. The primary aim of the article is to argue that this use of free recall is problematical, because it is based on an overly simplistic view of connectives. The main basis for the argumentation is recent linguistic research into the meaning and function of discourse connectives. It will also be argued that, given the insights yielded by such research into discourse connectives, an amended use of a measure of free recall could be a valuable tool for investigating the effect of discourse connectives on discourse comprehension. A 
secondary aim of the article, accordingly, is to argue that there are benefits to be gained from closer contact between discourse comprehension research and recent linguistic research.

In order to make the discussion more concrete, I will take as a reference point Roen's (1984) use of free recall as a measure of the effect of discourse connectives on discourse comprehension. What makes Roen's study an interesting focus for the argumentation is that he actually expresses some skepticism against the widely accepted "the-more-the-better" view on the use of discourse connectives. Note, though, that the argumentation presented below is not directed specifically and exclusively at Roen (1984). Rather, it is directed at a particular use of free recall as a measure of discourse comprehension, a use instantiated by Roen (1984), amongst others.

Discourse connectives and free recall: evidence for a negative effect on discourse comprehension?

Roen (1984) used written free recall (together with reading rate) to probe the effect of a variety of variables on discourse comprehension. Our concern is with the frequency of discourse connectives - which Roen refers to as "cohesive conjunctions" - in Roen's test passages. Roen used two passages - one dealing with the controversy over the evolutionary significance of Neanderthal man, and the other with the anthropological significance of a Pre-Neolithic farming village unearthed in Southeastern Asia. The text length varied between 745 and 921 words. Each passage was an example of fairly technical, social-scientific prose. Various versions of these passages were prepared, one set in terms of the level of discourse connectives used in the text. The discourse connective (=cohesive conjunction) high version contained 43 discourse connectives which did not occur in the discourse connective low version. In the discourse connective moderate version there appeared 19 discourse connectives which did not appear in the low version. All the discourse connectives in question functioned as intersentential connectives. That is, they made explicit the relationships between different sentences in the text. 
For one of the passages - the Pre-Neolithic Farming passage - the level of discourse connectives did not significantly affect written free recall. For the other passage - the Neanderthal passage - there was no significant difference in free recall for the middle and low versions. For the discourse connective high version, though, recall was significantly lower than for either the moderate or low versions.

The latter finding is surprising, if one assumes that discourse connectives make texts more readable, and that their presence in a text thus contributes positively to the comprehension of a text. Roen's finding that free recall for the discourse connective high version of the Neanderthal passage was adversely affected by a high number of discourse connectives is thus precisely the opposite of what one would expect. This finding forms the basis for Roen's skepticism towards the "the-more-the-better" view on the use of discourse connectives. If a high level of discourse connectives can actually lead to lower levels of free recall, then by implication higher levels of discourse connectives can adversely affect reading comprehension. Hence, the "the-more-the-better" advice could lead to less readable prose. To put it differently: Roen's findings would seem to suggest that the cause of effective expository writing may actually be undermined by the use of high levels of discourse connectives.

The crucial assumption underlying experimental studies such as Roen's can be formulated as follows: If discourse connectives facilitate discourse comprehension, then there should be a positive correlation between the level of discourse connectives in a text and the level of free recall for that text. Without this assumption, for instance, Roen has no basis for his skepticism about the unqualified advice on the use of discourse connectives. Moreover, the entire rationale for his experiment, and for other similar experiments which use free recall as a measure of the facilitative effect of discourse connectives on discourse comprehension, becomes suspect. It therefore seems worthwhile to examine the validity of this basic assumption underlying Roen's work. Recent linguistics research on discourse connectives - briefly outlined in the next section provides us with a basis for such an appraisal. 


\section{Discourse connectives as instructions for processing}

How should the meaning of discourse connectives be characterized? What is their function in discourse comprehension? While I do not wish to suggest that there is anything like consensus on these issues amongst linguists, there is nevertheless a considerable, and growing, body of linguistic work on discourse connectives that subscribes to the following two basic assumptions about such connectives:

(a) Discourse connectives do not encode concepts. That is, their meaning cannot be characterized in terms of some contribution which they make towards the content of the representations which an interpreter constructs in his mind on the basis of the utterance containing the connective.

(b) What discourse connectives do contribute to discourse comprehension, is a set of instructions for the processing of the representations constructed in the mind of the interpreter. That is, the meaning of discourse connectives is to be analyzed in procedural terms.

These basic assumptions about the meaning and function of discourse connectives are articulated and argued for in various ways by a variety of linguists, including pragmaticians, discourse analysts and psycholinguists (Fraser, 1990; Caron, 1987; 1992; Blakemore, 1987; 1988; 1989; 1990; 1992; Blass, 1990; Jucker, 1993; Haegeman, 1993). The assumption that the contribution of discourse connectives to discourse comprehension should be analyzed in $\stackrel{\Phi}{\omega}$ procedural terms also underlies the work done by Tyler and Bro on nonnative discourse (Tyler and Bro, 1992; 1993).

Caron's $(1987,1992)$ articulation of the procedural view of discourse connectives is particularly clear and unambiguous. According to him (1987: 577), connectives "do not give information (about states of things), but instructions for using information (according to the speaker's goals)." And he (1992: 167) states that certain linguistic markers - including 
connectives - "thus appear to act as processing instructions which order the way in which information is processed by the listener and integrated in the representation of the discourse..."

The most detailed account of the procedural nature of discourse connectives within pragmatics is developed within the framework of Sperber \& Wilson's (1986) relevance theory. Blakemore and Blass are prominent pragmaticians involved in this development. Relevance theory views linguistic comprehension as inferential comprehension, fed by linguistic decoding. Inferential comprehension, according to Wilson and Sperber (1993: 10, involves "the construction and manipulation of conceptual representations". Linguistic constructions, therefore, may be expected "to encode two basic types of information: concepts or conceptual representations on the one hand, and procedures for manipulating them on the other."

Discourse connectives such as so, after all, but are then analyzed as linguistic constructions which encode procedures for the manipulation of conceptual representations. For instance, so instructs the interpreter to process the proposition expressed by an utterance introduced by it in a context which will enable the interpreter to identify the proposition as a contextual effect of the preceding utterance.

While there undoubtedly are important differences among the various procedural views on the meaning and function of discourse connectives referred to above, the crucial point for the purposes of the current discussion is their agreement on the basic issue: Discourse connectives provide instructions to the interpreter's mind on how to process the information/conceptual representations constructed on the basis of the utterances in question. And indeed, the arguments put forward by the various authors in support of their procedural accounts of discourse connectives collectively provide strong support for this assumption about the meaning and function of connectives.

Note also that all these views define the meaning and function of discourse connectives in mentalistic terms, that is, with reference to the mental systems and processes involved in utterance interpretation. The significance of this point will become clearer below.

The assumption that the meaning and function of discourse connectives are to be analyzed in procedural terms can be integrated in an interesting way with another assumption about the function of such connectives in discourse. Chen (1991) argues that logical connectors - discourse 
connectives, in our terminology - have an important function in grounding the clause they introduce. Chen (1991: 434) bases his analysis of the grounding function of connectives on Hopper \& Thompson's (1980:280) and Wallace's (1982: 208) definitions of background and foreground. In terms of these definitions, the background includes information which "does not immediately and crucially contribute to the speaker's goal" (Hopper \& Thompson, 1980: 280), information which is of "lesser importance" (Wallace, 1982: 208). Foreground, by contrast, consists of the material which "supplies the main point of the discourse" (Hopper \& Thompson, 1980: 280), or the "more important" elements of the discourse (Wallace, 1982: 208). According to Chen, some connectives - e.g. though, although - always serve to background information. Others - such as but, yet, nevertheless - always foreground information.

Chen's views on the grounding function of discourse connectives can be accommodated straightforwardly on the assumption that the meaning of such connectives is to be analyzed in procedural terms. The grounding properties of a discourse connective are to be incorporated in the set of procedures which constitute the meaning of the connective, procedures which instruct the hearer/reader how to process the information conveyed by the utterance containing the connective. Such an integration of Chen's views on the grounding characteristics of discourse connectives with the procedural view of connectives is quite in line with his (1991: 443) own informal comments on the contribution of connectives to good writing. For instance, he (1991:443) claims, in a reference to some work by Celce-Murcia and Larsen-Freeman, that "the correct use of connectors can signal to the reader/listener what is the most important information, what is the skeleton of the discourse, thus guiding the reader/istener in the right direction in his/her comprehension [my emphasis - M.S.]." 
Free recall, discourse comprehension, and the processing instructions issued by discourse connectives

What are the implications of these assumptions about the meaning and function of discourse connectives for the use of free recall as a measure of the facilitative effect of connectives on discourse comprehension? If discourse connectives do indeed issue instructions on how to mentally process the information contained in utterances, then one would expect the memory representations of utterances containing such connectives to reflect these instructions. Moreover, if some of these processing instructions instruct the reader/listener to background or foreground some of the information expressed by an utterance, the memory representation of the utterances should also reflect these backgrounding and foregrounding instructions. Consequently, one would expect some of the information conveyed by a text containing backgrounding discourse connectives to be represented "less prominently" in memory than other information contained in the text. And by implication, not all information obtained through reading of the text should be equally accessible in free recall.

Looked at from this perspective, a lower level of recall for a text containing a high level of discourse connectives could in principle provide evidence for a positive effect of such connectives on comprehension, rather than the opposite. This means, for example, that Roen's (1984) findings on the negative correlation between the level of discourse connectives in a text and the level of free written recall for that text do not necessarily provide support for the view that the use of such connectives can adversely affect comprehension. Our current understanding of the meaning and function of discourse connectives suggests another possible explanation for Roen's findings: The connectives in Roen's text may have contained mental instructions for backgrounding. And if this is the case, Roen would no longer have a basis for his skepticism against the "the-more-the-better" advice on the use of discourse connectives.

The crucial question to ask about Roen's findings is obvious: What are the functions of the discourse connectives which appear in the discourse connective high version of Roen's test passage, but not in the moderate and low versions? Do these discourse connectives instruct the reader to background or foreground the relevant information? For Roen's interpretation of the 
negative correlation as indicative of a negative effect on comprehension to go through, these connectives would have to be foregrounding, and not backgrounding, connectives.

Unfortunately Roen's (1984) study does not include the different text versions used by him. Consequently, it is not possible to make the necessary checks. Given the assumptions about the functions of discourse connectives outlined above, Roen's failure to identify and analyze the discourse connectives involved in his experimental passages represents a serious shortcoming of this study.

The problem is not confined to Roen's study. Similar concerns arise over any study which uses free recall as a measure of the facilitative effect of discourse connectives on discourse comprehension, including those studies which claim a facilitative effect for discourse connectives. For instance, how should a higher level of free recall for sentences introduced by backgrounding connectives be interpreted? As evidence for or against a facilitating effect on discourse comprehension? To give us real insight into the effect of connectives on comprehension, free recall studies would have to consider not only the number of connectives appearing in a text, but also the precise nature of the instructions for processing encoded by these connectives. In particular, they will have to consider very carefully whether the connectives instruct the reader to background or foreground certain information. Only by adopting such a fine-grained approach to discourse connectives can free recall studies hope to throw light on the effect of discourse connectives on discourse comprehension.

Caron's (1987) study on the connection between the procedural meaning of discourse connectives and their effect on memory illustrates such a more fine-grained approach. Caron conducted an experiment basically along the same lines as Roen's. Two versions of a French argumentative passage were presented to two groups of subjects. The first group heard the original version of the passage, which contained a number of discourse connectives. The second group heard the same text, but with all connectives removed. Caron's findings showed that the discourse connectives had a differential effect on recall. Some discourse connectives gave rise to a better recall, while others reduced the level of recall. Caron (1987: 569-570) discusses two examples to substantiate his claim. 
Sentence 5 in the text was linked to the preceding sentence 4 by the discourse connective d'ailleurs (roughly translatable as "besides"). This connective introduces a fact which provides evidence for the argument expressed in the preceding sentence. Recall of sentence 5 was significantly better for the group who read the text version with connectives than for those who read the version without connectives. Moreover, the "connective" group more frequently recalled sentences 4 and 5 together.

This recall pattern contrasts with that for the last sentence in the text, sentence 10, which

in in the connective version was introduced by même si ("even if"). Without the discourse $\circ$ $\because$ connective, sentence 10 was significantly better recalled than sentence 9 - which Caron ascribes to the usual "recency effect". With the connective, though, the recall for sentence 10 became

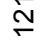

significantly poorer. That is, in this case the presence of the connective had a negative effect on 은 of free recall.

So, as Caron (1987: 571) puts it; "the mere presence of a connective can affect the क्ष presence of the discourse connective enhances recall; sometimes it does not. The interesting point noted by Caron is that the observed effect of the discourse connectives "were just what they had to be according to the speaker's goals". d'ailleurs (= "besides"), with the better recall, introduced a sentence which gave factual evidence for a claim the speaker has just made. même si (= "even if"), with the lower recall, marked the sentence as irrelevant for the main line of the argument. The linkup with Chen's views on the backgrounding and foregrounding of utterances by means of discourse connectives should be obvious. As Caron (1987: 571) puts it, we can say that the speaker, by means of the discourse connectives, gave the hearer instructions on how to process the information expressed in the text, instructions which, automatically executed, lead to a representation in memory consistent with the goals of discourse. 


\section{Conclusions}

The existence of Caron's (1987) study serves to strengthen the doubts that can be raised on theoretical linguistic grounds against Roen's (1984) study. Caron's (1987) work on the effect of discourse connectives on free recall underlines that a negative correlation between the level of ¿̂discourse connectives in a text and the level of free recall does not necessarily provide a basis for

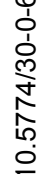
connectives. Given the procedural nature of discourse connectives, and given that they can encode instructions for the grounding of clauses, one would in fact expect some uses of б̈connectives to have a negative effect on free recall. Differing levels of recall for different $\underset{\sim}{\sim}$ utterances in a text, if correlated with the backgrounding or foregrounding function of the O․ discourse connectives introducing these utterances, would then provide supporting evidence for $\mathscr{O}_{0}$ the idea that such connectives facilitate discourse comprehension.

More generally, Caron's (1987) study provides a concrete illustration of the need for jresearchers investigating the link between the presence of discourse connectives and level of free ifrecall to use a more sophisticated conception of the meaning and function of discourse connectives. Such research must take into account not only the level of discourse use in a text, $\stackrel{\subseteq}{\exists}$ but also the specific contribution which each connective is intended to make to the interpretation $\subseteq$ on of the discourse.

ब(

Stated positively, the argumentation presented above suggests that recent linguistic ర్ research on the meaning and function of discourse connectives can provide empirical researchers 8

effect of discourse connectives on discourse comprehension were to take into account recent linguistic insights into the meaning and function of discourse connectives, they might be able to use free recall to develop a much clearer and more fine-grained account of the actual effect of such connectives on discourse comprehension. Caron's (1987) study is a case in point.

By the same token, the connections drawn above between linguistic claims about the meaning and function of discourse connectives and empirical research on the effect of 
connectives on memory representations suggest that linguistics too might benefit from taking note of empirical research into discourse comprehension. Insofar as pragmatics makes claims about the ways in which discourse connectives (or other linguistic elements) affect the mental processing of information conveyed by utterances, these claims should be subjected to empirical inquiry. Caron's (1987) study provides us with an illustration of how empirical research into O̊discourse comprehension can provide evidence for claims about the meaning and function of ìn Oprovide an important evidential base for the testing of pragmatic claims about the meaning and क्̈function of such connectives.

I would like to thank Henriëtte Murray for her constructive comments on an earlier version of this text.

के

wi** The work on which this article is based was completed while the author was attached to the

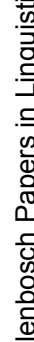
Department of General Linguistics, University of Stellenbosch.

REFERENCES

$\frac{\pi}{\circ}$

Blakemore, Diane. (1987). Semantic constraints on relevance. Oxford: Blackwell.

Blakemore, Diane. (1988). So as a constraint on relevance. In: R. Kempson (ed.), Mental representation: The interface between language and reality, 183-195. Cambridge: Cambridge University Press.

Blakemore, Diane. (1989). Denial and contrast: a relevance theoretic analysis of but. Linguistics and Philosophy 12: 15-38. 
Blakemore, Diane. (1990). Constraints on interpretation. Proceedings of the Sixteenth Annual Meeting of the Berkeley Linguistics Society: 363-370.

Blakemore, Diane. (1992). Understanding utterances. An introduction to pragmatics. Oxford: Blackwell.

Blass, Regina. (1990). Relevance relations in discourse. Cambridge: Cambridge University Press.

Bridge, Connie, \& Winograd, Peter N. (1982). Readers' awareness of cohesive relationships during cloze comprehension. Joumal of Reading Behavior XIV: 299-312.

Caron, Jean. (1987). Processing connectives and the pragmatics of discourse. In: J. Verschueren \& M. Bertuccelli-Papi (eds.), The pragmatic perspective (Selected papers from the 1985 Intemational Pragmatics Conference), 567-580. Amsterdam: Benjamins.

Caron, Jean. (1992). An introduction to psycholinguistics. New York etc.: Harvester.

Chen, Rong. (1992). Logical connectors, grounding and cognition. Language Science 13: 433445.

Fraser, Bruce. (1990). An approach to discourse markers. Journal of Pragmatics 14: 383-395.

Geva, Esther, \& Ryan, Ellen Bouchard. (1985). Joumal of Reading Behavior XVII: 331-346.

Haegeman, Liliane. (1993). The interpretation of the particle da in West Flemish. Lingua 90: 111-128. 
Halliday, M.A.K., \& Hasan, R. (1976). Cohesion in English. London: Longman.

Hopper, P., \& Thompson, S.A. (1980). Transitivity in grammar and discourse. Language 56: 251-299.

Irwin, Judith W. (1980). The effects of explicitness and clause order on the comprehension of reversible causal relationships. Reading Research Quarterly 4: 477-488.

Jucker, Andreas H. (1993). The discourse marker well: A relevance-theoretic account. Journal of Pragmatics 19: 435-452.

Marshall, N., \& Glock, M.D. (1987). Comprehension of connected discourse: A study into the relationships between the structure of text and information recalled. Reading Research Quarterly 14: 10-56.

Meyer, B.J.F., Brandt, D.M., \& Bluth, G.J. (1980). Use of top-level structure in text: Key for reading comprehension of ninth-grade students. Reading Research Quarterly 16: 72-102.

Roen, Duane H. (1984). The effects of cohesive conjunction, reference, response rhetorical predicates, and topic on reading rate and written free recall. Journal of Reading Behavior XVI: 15-26.

Sperber, Dan, \& Wilson, Deirdre. (1986). Relevance. Communication and cognition. Oxford: Blackwell.

Spyridakis, Jan H., \& Standal, Timothy C. (1987). Reading Research Quarterly XXII(3): 285298. 
Tyler, Andrea, \& Bro, John. (1992). Discourse structure in nonnative English discourse. The effect of ordering and interpretive cues on perceptions of comprehensibility. Studies in Second Language Acquisition 14: 71-86.

đ Tyler, Andrea, \& Bro, John. (1993). Discourse processing effort and perceptions of comprehensibility in nonnative discourse. The effect of ordering and interpretive cues revisited. Studies in Second Language Acquisition 15: 507-522.

Wallace, S. (1982). Figure and ground: the interrelationships of linguistic categories. In: P.J. Hopper (ed.), Tense-aspect: Between semantics and pragmatics, 201-223. Amsterdam: John Benjamins.

Williams, Ray. (1985). Readable writing. Harlow: Longman.

Wilson, Deirdre, \& Sperber, Dan. (1993). Linguistic form and relevance. Lingua 90: 1-25.

Zinar, Susan. (1990). Fifth-graders' recall of propositional content and causal relationships from expository prose. Journal of Reading Behavior XXII: 181-199. 